\title{
Successful treatment with lenalidomide dexamethasone for second relapse of a patient with non-secretory multiple myeloma with extramedullary liver plasmacytomas: A case report
}

Gavriatopoulou Maria*, Terpos Evangelos, Roussou Maria, Migkou Magdalini, Ntanasis-Stathopoulos Ioannis, Kastritis Efstathios and Meletios Athanasios Dimopoulos

Alexandra Hospital, Department of Clinical Therapeutics, Athens Medical School, National and Kapodistrian University of Athens, Greece

\begin{abstract}
MM is defined as the presence of $\geq 10 \%$ of monoclonal plasma cells (PCs) in the bone marrow (or a biopsy-proven extramedullary plasmacytoma) along with endorgan damage due to the underlying disorder. For the majority of myeloma patients, plasma cells are restricted in the bone marrow. However, a small subgroup of patients might develop extramedullary myeloma (EMM), which is defined as the presence of clonal PCs outside the bone marrow. Magnetic resonance imaging (MRI) and positron emission tomography/computed tomography (PET-CT) are the gold standard imaging techniques for the diagnosis, while biopsy of the lesion is highly recommended. The prognosis is very poor with a median overall survival that does not exceed 6 months. Patients with extramedullary disease are considered as high-risk patients and should be treated accordingly. In this case report we describe the management of a patient with nonsecretory myeloma who relapsed twice with extramedullary disease and we summarize the recommendations for therapeutic approaches of these patients that remain very challenging.
\end{abstract}

\section{Introduction}

Multiple myeloma (MM) is a plasma cell (PC) malignancy that accounts for approximately $10 \%$ of all hematologic malignancies [1]. Median age at the time of diagnosis varies from 63 to 70 years [2]. Over the past years, the median survival of myeloma patients has almost doubled [3] and this improvement is mostly due to the use of high-dose therapy followed by autologous stem cell transplantation (ASCT), in addition to novel agent's incorporation including proteasome inhibitors (PIs) and immunomodulatory drugs (IMiDs). More effective novel therapeutic strategies including next-generation IMiDs (pomalidomide), nextgeneration PIs (carfilzomib, ixazomib, oprozomib, and marizomib), histone deacetylase inhibitors (panobinostat), and monoclonal antibodies (daratumumab, isatuximab, MOR202 and elotuzumab) are currently approved or under investigation for the management of relapsed and/or refractory disease [4]. MM is defined as the presence of $\geq 10 \%$ of monoclonal plasma cells (PCs) in the bone marrow (or a biopsy-proven extramedullary plasmacytoma) along with endorgan damage which is attributed to the underlying disorder (anemia, hypercalcemia, renal insufficiency, and bone involvement) [5]. For the majority of myeloma patients, plasma cells are restricted only in the bone marrow. However, a small subgroup of patients might develop extramedullary myeloma (EMM), defined as the presence of clonal PCs outside the bone marrow [6]. Extramedullary disease is spreading hematogenously and refers to soft tissue infiltration by plasma cells (liver, CNS, skin, pancreas, lymph nodes, kidneys, pleural effusions) $[6,7]$. EMM is diagnosed at approximately $6-8 \%$ of the MM patients at the initial diagnosis while the prevalence increases in the relapsed/ refractory setting and reaches almost $10-30 \%$ of the patients [8-11]. Extramedullary plasmacytomas usually show a more aggressive, plasmablastic morphology [6]. High LDH levels, high-risk cytogenetic features, anemia, thrombocytopenia and nonsecretory MM are more frequent in patients with EMM [11-13]. Currently, the underlying molecular pathogenesis of the extramedullary spread of PCs is only partially understood. The presence of EMM at the initial diagnosis is correlated with poor prognosis $[11,14]$. However, at the time of relapse, extramedullary disease has even worse prognosis with an overall survival that does not exceed 6 months [15]. The diagnostic and therapeutic approach of EMM is very challenging especially due to the adverse prognostic features and heterogeneity. We describe here a patient with nonsecretory multiple myeloma who has progressed twice with extramedullary disease, he was successfully treated initially with bortezomib and subsequently with lenalidomide and he still remains in remission.

\section{Case presentation}

A 62-year-old man was diagnosed with non-secretory $\mathrm{MM}$ in 2007. At this time, he presented with symptomatic myeloma-related

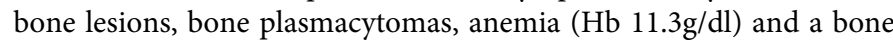
marrow biopsy confirmed the presence of 30\% PCs. Both serum and urine immunofixation were negative while the free light chain ratio was abnormal (6.08). The International Scoring System (ISS) staging was

Correspondence to: Gavriatopoulou Maria, Alexandra Hospital, Department of Clinical Therapeutics, Athens Medical School, National and Kapodistrian University of Athens, Greece, Tel: +306934137080; E-mail: mariagabria@gmail.com

Received: January 12, 2017; Accepted: February 04, 2017; Published: February 06,2017 
III, and a fluorescence in situ hybridization (FISH) analysis revealed $13 \mathrm{q}$ deletion and addition of 1q21 chromosome. The LDH was normal; thus, the R-ISS staging was II. The first line of therapy consisted of 4 cycles of bortezomib-adriamycin-dexamethasone (PAD regimen) followed by mobilization with cyclophosphamide and high-dose melphalan/ASCT. The patient achieved a complete remission (CR) after the completion of the induction therapy which was maintained for 3 years post transplantation. During this period the patient was treated only with zolendronic acid. Then, the patient relapsed with an increase of the kappa light chain $(139 \mathrm{mg} / \mathrm{L})$ but he remained asymtpomatic, therefore he was closely monitored but not treated. Eighteen months later the patient was admitted to our department due to fever of unknown origin. The patient was hospitalized and the abdomen CT scan performed revealed multiple hepatic lesions with a maximum diameter of $5,5 \mathrm{~cm}$ more compatible with radiological criteria with secondary metastatic lesions. The patient underwent biopsy under CT guidance and the pathology report revealed infiltration by monoclonal plasma cells. Bone marrow aspirate infiltration was $15 \%$. The patient was treated with a regimen consisting of bortezomibcyclophosphamide-dexamethasone (VCD) in which bortezomib was administered at a weekly basis for 8 cycles and then alone every other week thereafter until cycle 20 . At that time the patient underwent a scheduled follow-up MRI which revealed progressive disease with liver extramedullary plasmacytomas. Subsequently PET-CT was performed to confirm the diagnosis. No other abnormal radiological findings were noted. Bone marrow aspirate revealed $10 \%$ infiltration. The patient then started treatment with lenalidomide (25 mg/d, days 1-21) plus low-dose dexamethasone (40 mg/d, once weekly) (Len-Dex). The clinical evolution was favorable with complete disappearance of the lesions. The patient continues to receive Len-Dex therapy for 29 months and still remains in complete remission with negative serum and urine immunofixation, negative bone marrow biopsy, completely normal PET-CT and normal FLCs and FLCs ratio.

\section{Discussion}

This case report illustrates the diagnostic and therapeutic approach to an EMM relapse. This high-risk MM patient (stage 3 ISS) achieved a CR after the completion of an intensive approach combining bortezomib-based induction and high-dose melphalan. 4 years later, he presented with fever of unknown origin that did not initially suggest a MM relapse. However, clinical tests revealed liver tumor lesions, which were proven to be an extramedullary manifestation. In this context of possible EMM, MRI and PET-CT are valuable tools to detect lesions with increased FDG avidity [16]. In order to confirm the diagnosis a biopsy should be performed. Extramedullary relapse has extremely poor prognosis that does not exceed 6 months [15]. The therapeutic approach should take into account previous lines of therapy and duration of response. In the present case, we started a bortezomib-based triplet combination which maintained the patient in remission for 18 months. The patient progressed while on treatment with a PI based regimen therefore the combination of lenalidomide dexamethasone was initiated. The patient is still receiving treatment until today and remains in complete remission. Novel therapeutic approaches recently approved or currently investigated will probably improve response rates and overall survival for this group of patients. The identification of the underlying molecular pathophysiology in EMM might lead us to individualized and targeted therapies in order to overcome the adverse prognosis. Currently, according to the international guidelines, only plain radiography and MRI are recommended for the initial imaging staging $[16,17]$. However, PET-CT remains a very effective tool to detect extramedullary disease [18]. There is still lack of prospective clinical trials designed specifically for extramedullary disease. Therefore, it is difficult to recommend one therapeutic strategy over another. However, EMM is considered as high-risk disease; therefore, the therapeutic choices should be aggressive and intensive [19]. EMM patients at diagnosis if eligible should be treated with stem cell transplantation, following a triplet induction combination. Subsequently a triplet consolidation therapy, and maintenance treatment, should be administered. Tandem ASCT should be considered for ultra-high risk patients [20]. For elderly MM patients, not eligible for ASCT, bortezomib-melphalan-prednisone (VMP) or continuous Len-Dex seem to be two of the most effective combinations for up-front therapy. The role of monoclonal antibodies is still under investigation. For relapsed EMM patients previous lines of therapy and the duration of response should guide the therapeutic choices. Biopsy confirmation is highly encouraged and may provide the biological rationale for individualized targeted therapies. Immunotherapy and molecular therapies have shown quite encouraging results; however, data is still very limited [21-24]. In conclusion, EMM is an entity with poor prognosis that affects approximately $15 \%$ of the patients at some timepoint during the course of the disease. The efforts should focus on optimal detection of EMM and prospective clinical trials for this adverse prognostic group should be designed in the near future to help define the most appropriate therapeutic approach.

\section{References}

1. Becker N (2011) Epidemiology of multiple myeloma. Recent Results Cancer Res 183 25-35.

2. Moreau P, San Miguel J, Ludwig H (2013)Multiple Myeloma: ESMO Clinical Practice Guidelines. ESMO. Ann Oncol 24 (Suppl 6): vi133-vi137.

3. Kumar SK, Rajkumar SV, Dispenzieri A, Lacy MQ, Hayman SR, et al. (2008) Improved survival in multiple myeloma and the impact of novel therapies. Blood 111: 2516-2520.[Crossref]

4. Nooka AK, Kastritis E, Dimopoulos MA, Lonial S (2015) Treatment options for relapsed and refractory multiple myeloma. Blood 125: 3085-3099.[Crossref]

5. Rajkumar SV, Dimopoulos MA, Palumbo A, Blade J, Merlini G5, et al. (2014) International Myeloma Working Group updated criteria for the diagnosis of multiple myeloma. Lancet Oncol 15: e538-548.[Crossref]

6. Bladé J, Fernández de Larrea C, Rosiñol L (2011) Soft-tissue plasmacytomas in multiple myeloma: incidence, mechanisms of extramedullary spread, and treatment approach. J Clin Oncol 29: 3805-3812.

7. Weinstock M, Ghobrial IM (2013) Extramedullary multiple myeloma. Leuk Lymphoma 54: 1135-1141.[Crossref]

8. Zamagni E, Patriarca F, Nanni C (2011) Prognostic relevance of 18-F FDG PET $\mathrm{CT}$ in newly diagnosed multiple myeloma patients treated with up-front autologous transplantation. Blood 118: 5989-5995.

9. Bartel TB, Haessler J, Brown TLY (2009) F18-fluorodeoxyglucose positron emission tomography in the context of other imaging techniques and prognostic factors in multiple myeloma. Blood 114: 2068-2076.

10. Weinstock M, Aljawai Y, Morgan EA, Laubach J (2015) Incidence and clinica features of extramedullary multiple myeloma in patients who underwent stem cell transplantation. Br J Haematol 169: 851-858.[Crossref]

11. Varettoni M, Corso A, Pica G (2010) Incidence, presenting features and outcome of extramedullary disease in multiple myeloma: a longitudinal study on 1003 consecutive patients. Ann Oncol 21: 325-330.

12. Billecke L, Murga Penas EM, May AM, Engelhardt M, Nagler A, et al. (2013) Cytogenetics of extramedullary manifestations in multiple myeloma. Br J Haematol 161: 87-94.[Crossref]

13. Rasche L, Bernard C, Topp MS (2012) Features of extramedullary myeloma relapse: high proliferation, minimal marrow involvement, adverse cytogenetics: a retrospective single-center study of 24 cases. Ann Hematol 91: 1031-1037.

14. Usmani SZ, Heuck C, Mitchell A, Szymonifka J, Nair B, et al. (2012) Extramedullary 
Maria G (2017) Successful treatment with lenalidomide dexamethasone for second relapse of a patient with non-secretory multiple myeloma with extramedullary liver plasmacytomas: A case report

disease portends poor prognosis in multiple myeloma and is over-represented in highrisk disease even in the era of novel agents. Haematologica 97: 1761-1767.[Crossref]

15. Pour L, Sevcikova S, Greslikova H (2014) Soft-tissue extramedullary multiple myeloma prognosis is significantly worse in comparison to bone-related extramedullary relapse. Haematologica 99: 360-364.

16. Walker RC, Brown TL, Jones-Jackson LB, De Blanche L, Bartel T (2012) Imaging of multiple myeloma and related plasma cell dyscrasias. J Nucl Med 53: 1091-1101. [Crossref]

17. Dimopoulos MA, Hillengass J, Usmani S, Zamagni E, Lentzsch S, et al. (2015) Role of magnetic resonance imaging in the management of patients with multiple myeloma: a consensus statement. J Clin Oncol 33: 657-664.[Crossref]

18. Zamagni E, Nanni C, Mancuso K (2015) PET/CT improves the definition of complete response and allows to detect otherwise unidentifiable skeletal progression in multiple myeloma. Clin Cancer Res 21: 4384-4390.
19. Lonial S, Boise LH, Kaufman J (2015) How I treat high-risk myeloma. Blood 126: 1536-1543.[Crossref]

20. Shaughnessy JD, Zhou Y, Haessler J, van Rhee F, Anaissie E, et al. (2009) TP53 deletion is not an adverse feature in multiple myeloma treated with total therapy $3 . \mathrm{Br}$ J Haematol 147: 347-351.[Crossref]

21. Andrulis M, Lehners N, Capper D, Penzel R, Heining C, et al. (2013) Targeting the BRAF V600E mutation in multiple myeloma. Cancer Discov 3: 862-869.[Crossref]

22. Heuck C, Jethava Y, Khan RZ (2014) Targeted MEK inhibition in patients with previously treated multiple myeloma. Blood 124: 4775.

23. Garfall AL, Maus MV, Hwang WT, Lacey SF, Mahnke YD, et al. (2015) Chimeric Antigen Receptor T Cells against CD19 for Multiple Myeloma. N Engl J Med 373 : 1040-1047.[Crossref]

24. Rapoport AP, Stadtmauer EA, Binder-Scholl GK (2015) NY-ESO-1-specific TCRengineered $\mathrm{T}$ cells mediate sustained antigen-specific antitumor effects in myeloma. Nat Med 21: 914-921.

Copyright: (C2017 Maria G. This is an open-access article distributed under the terms of the Creative Commons Attribution License, which permits unrestricted use, distribution, and reproduction in any medium, provided the original author and source are credited. 